\title{
Higher desirability in solving multiple response optimization problems with committee machine
}

\begin{abstract}
Many industrial problems need to be optimized several responses simultaneously. These problems are named multiple response optimization (MRO) and they can have different objectives such as Target, Minimization or Maximization. Committee machine (CM) as a set of some experts such as some artificial neural networks (ANNs) in combination with genetic algorithm (GA) is applied for modeling and optimization of MRO problems. In addition, optimization usually is done on Global Desirability (GD) function. Current article is a development for recent authors' work to determine economic run number for application of $\mathrm{CM}$ and GA in MRO problem solving. This study includes a committee machine with four different ANNs. The CM weights are determined with GA which its fitness function is minimizing the RMSE. Then, another GA specifies the final solution with object maximizing the global desirability. This algorithm was implemented on five case studies and the results represent the algorithm can get higher global desirability by repeating the runs and economic run number (ERN) depends on the MRO problem objective. ERN is ten for objective ñTargetò. This number for objectives which are mixture of minimization and maximization ERN is five. The repetition are continued until these ERN values have considerable increased in maximum GD with respect to average value of GD. More repetition from these ERN to forty five numbers cause a slight raise in maximum GD.
\end{abstract}

Keyword: Economic run number; Global desirability; Committee machine; Multiple responses optimization 